OPEN ACCESS

Edited by:

Niccolo Terrando,

Duke University, United States

Reviewed by:

Valerio Chiurchiù,

Italian National Research Council, Italy

Dipak Panigrahy,

Harvard Medical School, United States

Nan Chiang,

Brigham and Women's Hospital and

Harvard Medical School, United States

*Correspondence:

Panagiotis F. Christopoulos christopoulos.panagiotis@rr-

research.no

Specialty section: This article was submitted to Inflammation,

a section of the journa

Frontiers in Immunology

Received: 22 November 2020 Accepted: 18 January 2021

Published: 24 February 2021

Citation:

Balta MG, Papathanasiou E and Christopoulos PF (2021) Specialized Pro-Resolving Mediators as Potential

Regulators of Inflammatory Macrophage Responses in COVID-19.

Front. Immunol. 12:632238. doi: 10.3389/fimmu.2021.632238

\section{Specialized Pro-Resolving Mediators as Potential Regulators of Inflammatory Macrophage Responses in COVID-19}

\author{
Maria G. Balta ${ }^{1}$, Evangelos Papathanasiou ${ }^{2,3}$ and Panagiotis F. Christopoulos ${ }^{4 *}$ \\ ${ }^{1}$ The CrossTalk Group, Institute of Oral Biology, University of Oslo, Oslo, Nonway, ${ }^{2}$ Department of Periodontology, Tufts \\ University School of Dental Medicine, Boston, MA, United States, ${ }^{3}$ Center for Clinical and Translational Research, Forsyth \\ Institute, Cambridge, MA, United States, ${ }^{4}$ Department of Pathology, Oslo University Hospital, Oslo, Norway
}

The recent outbreak of SARS-CoV2 has emerged as one of the biggest pandemics of our century, with outrageous health, social and economic consequences globally. Macrophages may lay in the center of COVID-19 pathogenesis and lethality and treatment of the macrophage-induced cytokine storm has emerged as essential. Specialized pro-resolving mediators (SPMs) hold strong therapeutic potentials in the management of COVID-19 as they can regulate macrophage infiltration and cytokine production but also promote a pro-resolving macrophage phenotype. In this review, we discuss the homeostatic functions of SPMs acting directly on macrophages on various levels, towards the resolution of inflammation. Moreover, we address the molecular events that link the lipid mediators with COVID-19 severity and discuss the clinical potentials of SPMs in COVID-19 immunotherapeutics.

Keywords: resolution of inflammation, lipid mediators/specialized pro-resolving mediators (SPMs), cytokine storm, macrophage activation syndrome (MAS), severe acute respiratory syndrome corona virus (SARS-CoV2)

\section{INTRODUCTION}

The recent outbreak of the corona virus disease 2019 (COVID-19) from Wuhan, China (1) has evolved to one of the biggest global pandemics of the 21st century. The infectious virus named by the World Health Organization as severe acute respiratory syndrome corona virus (SARS-CoV2) counts up to date (November 2020) more than 55.6 million people infected and 1.34 million deaths, since the start of the pandemic. According to the latest knowledge, disease pathogenesis is driven by a dysregulated immune response against SARS-CoV2, characterized among others by impaired type

Abbreviations: ALOX5, 5-lipoxygenase; ALOX15, 15-lipoxygenase; ARDS, Acute respiratory distress syndrome; (AT)-Rv, aspirin-triggered resolving; CCL, Chemokine (C-C motif) ligand; COVID-19, Corona virus disease 2019; CXCL, Chemokine (C-X-C motif) ligand; DHA, Docosahexaenoic acid; EPA, Eicosapentaenoic acid; IFN, Interferon; IL, Interleukin; LPS, Lipopolysaccharide; $\mathrm{LTB}_{4}$, Leukotriene B4; MaR, Maresin; MAS, Macrophage activation syndrome; NET, Neutrophil extracellular trap; NF-אB, Nuclear factor- $\mathrm{KB}$; NLRs, NOD-like receptors; PMN, Polymorphonuclear leukocyte; PRRs, Pattern recognition receptors; PTX3, Long pentraxin 3; PUFAs, Polyunsaturated fatty acids; Rv, Resolvin; RLRs, RIG-1 like receptors; SARS-CoV2, Severe acute respiratory syndrome corona virus; SPMs, Specialized pro-resolving mediators; TLRs, Toll-like receptors; TNF $\alpha$, Tumor necrosis factor $\alpha$. 
I IFN production, sustained inflammation and aberrant cytokine production $(2,3)$ similar to the "cytokine storm" syndrome that can become life-threatening. The consequences of this hyper immuno-activation, lead eventually to the clinical manifestations of severe COVID-19 including respiratory failure, systemic inflammation, acute respiratory distress syndrome (ARDS), multi-system inflammatory syndrome and multi-organ fibrosis and malfunction (4).

It is currently believed that alveolar macrophages may lay in the center of COVID-19 pathogenesis and lethality. SARS-CoV2 infection in the lungs and failure of rapid virus clearance, leads to severe inflammation as well as, tissue damage and fibrosis. This prolonged overactivation of resident macrophages by SARS$\mathrm{CoV} 2$ and augmented release of pro-inflammatory cytokines in the circulation, results in clinical manifestations similar to those described in the macrophage activation syndrome (MAS) (5). Therefore, identifying the factors that will regulate the macrophage responses in severe COVID-19 cases is essential for the disease recovery. In this review, we discuss the role of Specialized Pro-resolving lipid Mediators (SPMs) in driving the macrophages immuno-functions and thus regulating the macrophage-induced inflammation. Moreover, we address the molecular events that relate the lipid mediators with the disease pathology and discuss the clinical potentials of SPMs in COVID-19 treatment.

\section{MACROPHAGES AS KEY ORCHESTRATORS OF THE INFLAMMATORY MILIEU DURING CORONAVIRUSES INFECTIONS}

Macrophages represent an immune cell type of the myeloid lineage with broad spectrum of functions including among others phagocytosis of pathogens and debris, secretion of reactive species and cytokines, as well as, matrix remodeling and tissue repair $(6,7)$. Although they are considered as part of the innate immune system, they are known to bridge an adaptive immune response via presentation of antigens to $\mathrm{T}$ cells and activation of the latter in the tissues, via expression of co-stimulatory molecules and secretion of cytokines $(6,7)$. Recent evidence is also suggestive of the existence of memory macrophage populations driven by epigenetic modifications events, described under the term "trained immunity" (8). These exact "innate memory" properties of macrophages are currently under investigation in COVID-19 immunotherapeutics (9). Traditionally, the functional heterogeneity of macrophages is represented in vitro by a rather oversimplified model, dividing macrophages into two main phenotypes (M1 and M2). M1 phenotype refers to proinflammatory or classical activated macrophages, associated with Th1 responses and IFN $\gamma$ and/or LPS activation, while the M2 phenotype refers to anti-inflammatory or alternatively activated macrophages, associated with Th2 responses and IL-4 and/or IL-13 activation $(10,11)$. The terms "killing" or "healing" are also regularly used to describe M1 or M2 populations respectively. However, in vivo, there is not a clear cut off between these phenotypes but rather a continuum of functional states $(11,12)$ with M1 and M2 representing the opposing extremes of activation, thus delineating the need for reassessment of the current M1/M2 definition (13). Aiming for a lingua franca, a recent macrophage nomenclature has recommended the latest guidelines for macrophages activation, including the usage of the activator used in vitro instead of the typical M1 or M2 [e.g., $M(\operatorname{IFN} \gamma)$ ] and the combinational employment of multiple membrane markers as well as, transcription factors for phenotypic characterization of macrophages subtypes (14).

Along with neutrophils, macrophages constitute the first line of immune defense against microbial or viral infections. Invader sensing via a vast variety of pattern recognition receptors (PRRs), including Toll-like receptors (TLRs), RIG-1 like receptors (RLRs) and NOD-like receptors (NLRs) (15), located either on the cell surface or in intracellular compartments, leads to macrophage phenotype shift and pro-inflammatory activation. The single strand RNA receptor TLR7 has been addressed as a sensor for SARS-CoV2 (16). Of note, loss of function studies of TLR7 showed correlation with disease severity and dysregulated IFN type I response (17). Activation of the RNA sensing receptors like the TLR7 and TLR8 triggers downstream signaling pathways like NF$\mathrm{\kappa B}$ which in turn leads to transcription of various pro-inflammatory cytokines e.g., IL-1 $\beta$, TNF $\alpha$, IL-6, known as the first cytokine wave [reviewed in (18)]. A delayed IFN type I response, either due to genetic reasons or via direct viral-induced immuno-suppressive mechanisms (19-22), leads to impaired virus control and increased accumulation of inflammatory monocytes and macrophages with negative effects on T cells, as shown in a mouse model of SARS-CoV infection (23). Paradoxically this hyperactivation was induced by the type I IFN per se, pointing to the importance of timing for an efficient clearance of the virus. In any case, an increasing viral load results in continuously elevating numbers of activated macrophages and sustained hyperinflammation-known as the second cytokine wave-with severe tissue damage and life-threatening consequences [reviewed in (18)]. Very recently, several molecules associated with macrophages functions, such as the plasma levels of long pentraxin 3 (PTX3), as well as, the ratio of the transcription factors MAFB/ MAF have been suggested as potential prognostic indicators of disease progression and severity $(24,25)$. Of note, it has been speculated that the differences in disease severity between adults and children might be explained by the potential age-heterogeneity of macrophage populations in lungs and other organs during development (26) as well as, by the potential development of memory macrophages upon frequent adjuvant-involving vaccination during childhood (27).

Single-cell analysis has identified an $\mathrm{FCN1}^{+}$inflammatory macrophage phenotype in critical COVID-19 cases expressing the pro-inflammatory mediators IL-1 $\beta$, IL-8, TNF, CCL2, CCL3, CCL20, CXCL1, CXCL3, and CXCL10 (28). It is believed that this macrophage population actively regulates disease progression and fuels inflammation by continuously recruiting monocytes from the circulation and driving their differentiation. Same cytokines were also found in plasma from deceased COVID-19 patients (21). Similarly, the bronchoalveolar lavage fluid (BALF) from patients 
with severe COVID-19 was found rich in pro-inflammatory monocyte-derived macrophages (29). Moreover, lung macrophages from severe disease were found to produce higher levels of inflammatory cytokines (IL-6, IL-1 $\beta$ ) as well as, monocyte and neutrophil chemoattractants (IL-8, CCL2, CCL3, CCL4, CCL7), whereas macrophages from moderate COVID-19 patients produced higher volumes of the T-cell chemokine CXCL16 (29). Indeed, hypercytokinemia is considered a hallmark of the disease, while the serum levels can differentiate the disease severity (30). The direct implication of the cytokines involved in disease pathophysiology and the associated pneumonia, found both in MAS and in COVID-19, is highlighted in preliminary trials against SARS-CoV2, where blockade of IL-6 with tocilizumab showed promising clinical efficacy (31). However, whether IL-6 is detrimental or beneficial in COVID-19 remains unclear and is highly probable that timing is the most important factor in determining the success or not of anti-IL-6 treatments. Blockade of IL- 6 at the early stages of the disease development might negatively affect effective virus clearance [reviewed in (32)].

To this extent, treating the inflammation and the cytokine storm has emerged as a legitimate approach in COVID-19 therapeutics and appears to be equally important to anti-viral therapies. Various strategies have been proposed in this vein, including cytokine inhibitors (e.g., tocilizumab), corticosteroids, intravenous immunoglobulin (IVIG) and cytokine absorption devices [reviewed in $(18,33)]$. Since macrophages may also stand out as the key cell population in the resolution of inflammation and healing of the damaged tissue, identifying the factors inducing their phenotypic shift from the "pathologic" phenotype to the "healing" one, is essential in COVID-19.

\section{SPMS IN INFLAMMATION AND COVID-19}

Specialized pro-resolving mediators (SPMs) — consisting of lipoxins, resolvins, maresins, and protectins-represent a novel class of bioactive lipids that are generated by enzymatic oxygenation of n3 and n- 6 polyunsaturated fatty acids (PUFAs) after the initial stages of the inflammatory cascade (34). Endogenous biosynthesis of SPMs involves cell-cell interactions and is mediated by lipoxygenases (35). As the acute inflammatory response matures, accumulation of cells containing lipoxygenases (LOs) and corresponding pro-inflammatory products, such as prostaglandins (PGs), leukotrienes (LTs) and hydroxy acids (HETES), favors the "lipid mediator class switching". This phenomenon, as was initially described in neutrophils gives rise to the synthesis of SPMs through pathways that are spatially and temporally distinct from those involved in the generation of pro-inflammatory lipid mediators $(36,37)$. Lipoxins are synthesized through a series of enzymatic reactions starting with the oxidation of arachidonic acid (AA) by 15-LO through the process of transcellular biosynthesis, resulting in 15-S-hyroxy-(p)-eicosatetraenoic acid' [15-S-H(p)ETE]. Accordingly, 15-S-H(p)ETE is further acted on by $5-\mathrm{LO}$ to generate lipoxins, such as lipoxins $\mathrm{A}_{4}\left(\mathrm{LXA}_{4}\right)$ and $\mathrm{B}_{4}\left(\mathrm{LXB}_{4}\right)$ (36, 37). Similar effects can be reproduced by exogenous administration of low-dose aspirin, which acetylates cyclooxygenase-2 via 15R-LO, promoting the biosynthesis of epimeric (aspirin-triggered) (AT) forms of SPMs (15-epi-LXs or ATLs) (38).

E-series resolvins are produced by vascular endothelium via aspirin-modified COX-2 that converts eicosapentaenoic acid (EPA) to $18 \mathrm{R}$-hydro-peroxyeicosapentaenoic acid (18R-HEPE) and 18Shydro-peroxyeicosapentaenoic acid (18S-HEPE). These intermediates are rapidly taken up by human neutrophils and are metabolized to resolvin E1 (RvE1) and RvE2 by 5-LO. RvE1 biosynthesis can also be initiated by microbial cytochrome P450 mono-oxygenase in an aspirin-independent manner, which can contribute to its production in vivo $(39,40)$. D-series resolvins and protectins derive from docosahexaenoic acid (DHA) via subsequent 15-LO and 5-LO-mediated actions, while maresins are generated through 12-LO-mediated pathways (41). Thus, even though qualitatively the same enzymes are involved in either SPMs or eicosanoid biosynthesis, the exact molecular and biochemical mechanisms driving temporal relationships during the lipid mediator class switching remain to be further elucidated.

The balance of PUFAs-derived mediators in leukocytes has been associated with the localization of 5-LO (42). In fact, studies on macrophages in heightened inflammatory lesions such as advanced atherosclerotic plaques described abundant nuclear localization of 5-LO. Nuclear 5-LO because of its proximity to LTA4 hydrolase, seems to promote the conversion of AA to pro-inflammatory LTs in macrophages (43). On the contrary, non-nuclear localization of 5$\mathrm{LO}$, possibly due to its proximity to $12 / 15-\mathrm{LO}$, may favor the conversion of $\mathrm{AA}$ or $\mathrm{DHA}$ to lipoxins or $\mathrm{D}$-series resolvins respectively and has been therefore linked with enhanced SPMs formation (42-44). Interestingly, RvD1 has been described to shift 5-LO from the nucleus to the cytoplasm inducing a negative feedback loop that suppresses $\mathrm{LTB}_{4}$ formation and promotes generation of $\mathrm{LXA}_{4}$ in macrophages (44). In addition to the relocalization events, lipid mediator biosynthesis may be also affected by post-translational modifications, including miRNAs. For instance, increased expression of miR-466I in macrophages at early stages of inflammation promoted the synthesis of RvD1 in macrophages and the resolution of inflammatory exudates in mice (45). The regulation of resolution of inflammation by miRNAs via increasing the synthesis of SPMs and mediating resolution-phase macrophage polarizations needs to be further investigated. Once generated, SPMs act as agonists at specific G-protein-coupled receptors expressed on various cells including monocytes and macrophages and activate the resolution of inflammation, without causing systemic immunosuppression (46).

Among these receptors is ALX/FPR2, which apart from SPMs binds also protein ligands, such as, the acute-phase protein serum amyloid A (SAA) (47). Recently, SAA plasma levels were found to be dynamically increased with COVID-19 disease severity and this protein has been therefore proposed as a biomarker indicative of the severity and prognosis of the disease (48). Of note, SAA can antagonize the signaling of $\mathrm{LXA}_{4}$ and vice versa through allosteric inhibition of the receptor, inducing opposite intracellular effects (47). In fact, SAA inhibited the $\mathrm{LXA}_{4}$-mediated protective signaling in patients with chronic obstructive pulmonary disease resulting in defected activation of anti-inflammatory circuits providing a molecular explanation for SAA-mediated impaired resolution (49). 
Apart from SAA overexpression, COVID-19 has been characterized by elevated production of macrophage-derived eicosanoids that further enhance inflammation (50). In addition, a recent lipidomic analysis study showed a significant shift in the profile of lipid mediators with increased levels of arachidonate-derived proinflammatory lipid mediators (prostaglandins) in the sera of COVID-19 patients (51). Grouping of lipid mediators according to the oxygenasemediated synthesis pathway, demonstrated a greater activity of ALOX5, ALOX15, and cytochrome p450 (CYP) enzymes in the severe group (51). Further examination on the cell types responsible for the addressed lipidomic imbalance in this severe COVID-19 group, alluded neutrophils, as well as, a trend for CD14+, and CD16+ monocytes. Elevated ALOX5 expressing monocyte/macrophage populations have also been associated with severe disease (51). Similarly, increased ALOX5 activity related to symptom severity has been addressed in other viral infections e.g., in influenza (52).

Recently, COVID-19 has been also linked to dyslipidemia associated with deficiency of apolipoprotein E (ApoE) (50). ApoE is generated among other cells by lung macrophages and alveolar epithelial cells and exerts its protective effects by downregulating VCAM-1, inducing NO synthesis, inhibiting endothelial activation, and decreasing adhesion of monocytes to the endothelium (53). Apo: ${ }^{-/-}$mice under an omega-3 fatty acid-deficient diet presented significant overexpression of proinflammatory eicosanoids in the lungs as well as endothelial dysfunction which can contribute to increased blood coagulation found in severe COVID-19 (54). It has been therefore suggested that deficiency in ApoE found in SARS-CoV2 dyslipidemia may be linked to disease progression and complications (50). Of note, supplementation with EPA and DHA downregulated the levels of pro-inflammatory eicosanoids such as thromboxane $\mathrm{B} 2$ in the lungs of $\mathrm{ApoE}^{-/-}$mice and the effect was even more pronounced with the addition of aspirin treatment (54).

Known risk factors for severe COVID-19, including diabetes, obesity and chronic obstructive pulmonary disease (COPD), have been also related to dysregulated concentrations of SPMs $(55,56)$. Collectively these findings underscore the importance of fine-tuned lipid mediators' responses to achieve resolution in inflammationassociated pathologies. Severe COVID-19 may be characterized among others by a lipidomic imbalance in key cell populations contributing to the disease progression, such as neutrophils and/or monocytes/macrophages. As such, understanding the full antiinflammatory and pro-resolving spectrum of these lipid mediators is of major importance in the current coronaviruses pandemics.

\section{SPMS IN REGULATING MACROPHAGE IMMUNO-FUNCTIONS: MACROPHAGE INFILTRATION AND MOBILITY}

Recent evidence suggests that severe clinical manifestations found in COVID-19 are associated with excessive infiltration of inflammatory monocytes in the lungs, in the expense of tissueresident alveolar macrophages (29) and the subsequent release of pro-inflammatory cytokines in the circulation (5). Moreover, a population of these infiltrated monocyte-derived macrophages may be associated with the pulmonary fibrosis $(57,58)$, also found in progressed COVID-19 cases. Following a gradient of chemoattractant molecules and cytokines, circulating monocytes massively infiltrate the lungs, kidneys and other organs. In this vein, SPMs have been shown to inhibit monocyte recruitment into the tissues by regulating the leukocyte-endothelial interactions. Exogenous addition of resolvin E1 (RvE1) in whole blood was found to downregulate monocyte surface expression of adhesion molecules, mediating both early activation and rolling (i.e. Lselectin), as well as subsequent stable adhesion on the endothelium and transmigration into the tissues (e.g., CD18 integrin) (59). Incubation with RvE1 reduced the migration of M1-like macrophages (activated with LPS) towards the chemotactic protein chemerin, concomitant with a downregulation in the expression of ChemR23 receptor on macrophage surface, pointing to the regulatory role of this receptor in migration of macrophages to the inflamed area (60). Local application with RvE1 also decreased the numbers of infiltrating neutrophils, as well as, Th1 and Th17 cells in the cornea of mice with HSV-1-induced stromal keratitis (61). Similarly, other SPMs ( $\mathrm{LXA}_{4}$ and RvD1), in solution or incorporated into nano-proresolving medicines, reduced monocyte trafficking toward $\mathrm{LTB}_{4}$ (62). Of note, in a bacterial and viral lung co-infection model with robust recruitment of infiltrating monocytes and increased counts of exudative macrophages, exogenous delivery of AT-RvD1 during the acute phase of infection (day 4-6 post-pneumococcal inoculation), resulted in $\sim 50 \%$ reduction in infiltrating monocytes/macrophage numbers $\left(\mathrm{CD} 11 b^{\mathrm{Hi}}, \mathrm{CD} 11 c^{\mathrm{Low}}\right)$ (63). Since transcript or protein levels of key cytokines and chemokines associated with monocyte recruitment such as IL-1 $\beta$, IL-6, CCL2 and monocyte chemoattractant protein-1 (MCP-1) were not significantly reduced by this SPM, the authors suggested that AT-RvD1 directly affected the chemotactic properties of inflammatory monocytes (from the infected bronchioles to the distal lung alveoli) per se (63). In addition, halting further monocyte recruitment could prevent a monocyte-induced secondary phase of neutrophil migration into the lungs that would perpetuate acute lung injury. Interestingly, treatment with AT-RvD1 did not reduce exudative macrophages $\left(\mathrm{CD} 11 b^{\mathrm{Hi}}, \mathrm{CD} 11 c^{\mathrm{Hi}}\right)$ (63), a subpopulation of particular importance in the resolution processes represented by their ability to clear apoptotic neutrophils and facilitate the return to tissue homeostasis (64). Thus, SPMs may hold promising therapeutic potentials in decelerating the pathologic macrophage infiltration in inflamed lungs, during COVID-19 development.

\section{SPMS IN REGULATING THE CYTOKINE/ CHEMOKINE EXPRESSION OF MONOCYTES/MACROPHAGES}

A growing body of evidence points to the regulatory role of SPMs in pro-inflammatory cytokine secretion by a variety of immune cells, including monocytes and macrophages. SPMs inhibit the 
secretion of TNF, IL-1 $\beta$, IL- 8 by activating the GSK3 $\beta$ antiinflammatory axis in LPS-stimulated primary human monocytes (65). Resolvins inhibit the production of TNF- $\alpha$ and IL-6 by alveolar macrophages derived from both COPD and non-COPD individuals, although with a stronger potency in the latter (56). In addition, AT forms of SPMs were shown to decrease the secretion of macrophage migration inhibitory factor (MIF), plasminogen activator inhibitor-1 (PAI-1) and chemokine CCL2 in human monocyte-derived macrophages (66). Research focus on SPMs has recently resulted in the identification of novel peptide-containing conjugates with docosahexaenoic acid (DHA)-derived backbones. Resolvin peptide-containing conjugates in tissue regeneration (RCTRs), down-regulate inflammatory chemokines CXCL9 and CCL7 and increase IL-10 in human macrophages (67). Thus, in addition to the downregulation of pro-inflammatory molecules, SPMs and SPMs-peptide conjugates stimulate the production of antiinflammatory cytokines by macrophages such as IL-10 and TGF- $\beta$ (56), as well as of molecules associated with alternative macrophage activation (M2) and tissue healing e.g., IL-4, IL-11, and TGF- $\alpha$ (67). Physiologically, human macrophages may respond to different pathogens differently, as regards the lipid mediators' synthesis, depending on their activation phenotype. It was shown that Escherichia coli and Staphylococcus aureus stimulated the production of $\mathrm{LTB}_{4}$ and $\mathrm{PGE}_{2}$ in $\mathrm{M} 1$ macrophages, while favored the secretion of SPMs, including RvD2, RvD5 and maresin-1 in M2 macrophages (68). Mechanistically, SPMs act by regulating a variety of downstream signaling pathways including: both canonical and alternative NF$\mathrm{kB}$ pathways, STAT3, cAMP response element binding protein and MAPK signaling pathways $(56,69)$. In vivo evidence is also not lacking; RvD1 downregulates various pro-inflammatory cytokines and chemokines including TNF- $\alpha$, IL-6, CCL2, and IL- $1 \beta$ in peritoneal macrophages in mice (70). Of note, the involvement of macrophages in COVID-19 and the therapeutic potential of SPMs in the disease is further highlighted in a recent study; short incubation (3h) of monocyte-derived macrophages isolated from cystic fibrosis (CF) individuals with S1, S2 and N proteins from SARS-CoV-2 resulted in rapid release of chemokines such as IL-8 in cell-free supernatants (71). Most importantly, treatment with SPMs of CF macrophages stimulated with S1 protein resulted in a significant reduction in the release of IL-8, MCP-1 and macrophage inflammatory protein MIP- $1 \alpha$ (CCL3) and MIP-1 $\beta$ (CCL4) (71). Thus, treating the macrophage-generated cytokine storm in severe COVID-19 with SPMs, has emerged as a legitimate approach.

\section{SPMS IN REGULATING THE MACROPHAGE-MEDIATED EFFEROCYTOSIS AND THROMBUS RESOLUTION}

As noted above, clearance of dead cells and debris by professional phagocytes in the inflamed area-following the removal of the pathogen-is essential part of the resolution process. In fact, the uptake of dead cells (efferocytosis) per se or the extracellular vesicles secreted by dead cells, drive the reprogramming of macrophages towards an anti-inflammatory state (72-74). Macrophages play a critical role in orchestrating infectious inflammation towards its resolution (68). Accumulating evidence has documented a significant increase in macrophage uptake of apoptotic cells, following in vitro stimulation with SPMs, underlining their role as potent enhancers of macrophage efferocytosis $(75,76)$. Inflammation is characterized by a hypoxic milieu that develops, in part, via increased oxygen consumption by infiltrating leukocytes (77). SPMs promoted macrophage efferocytosis of neutrophils and erythrocytes in hemorrhagic exudates under hypoxic conditions in vivo. Of note, the engulfment of apoptotic PMNs and PMN microparticles by macrophages during efferocytosis increases the autocrine SPMs biosynthesis (78). In this vein, uptake of apoptotic cells by M1 macrophages (activated with IFN $\gamma+$ LPS) induced the production of SPMs, while suppressed prostanoids and leukotrienes, suggesting the presence of an endogenous negative feedback loop (79). In addition, aspirin-triggered forms of SPMs identified in patients with coronary artery disease receiving n-3 PUFA supplementation, significantly upregulated macrophage phagocytosis of blood clots, thereby promoting clot removal (80). Given the high risk for thrombosis found in severe COVID19 this may represent an additional protective function of SPMs related to their regulatory role on macrophages. Moreover, SARS-CoV2 was recently shown to stimulate the formation of neutrophil extracellular traps (NETs) in human neutrophils. The activation and release of NETs (NETosis) was found to be associated with increased levels of intracellular Reactive Oxygen Species (ROS) in these cells and was suggested to play an important role in thrombosis formation in COVID-19 patients (81). Interestingly, in vivo treatment with SPMs in mice was recently described to inhibit NETosis, indicating another protective effect of SPMs in thrombus resolution that could be of particular importance for COVID-19 $(82,83)$. SPMs may therefore have a beneficial effect on different cell types against COVID-19, towards the removal of dead cells and blood clots, as well as the resolution of inflammation and thrombroinflammation.

\section{SPMS IN REGULATING THE MACROPHAGE POLARIZATION}

Given the accumulating number of inflammatory macrophages infiltrating into lungs and other tissues and the natural plasticity of macrophages, a phenotypic shift of this pathogenic population may hold strong therapeutic potentials in COVID-19. Recent findings underlined the potential of SPMs to induce changes in macrophage phenotype towards a resolving profile, as indicated by a significant downregulation in the expression of M1associated markers (CD54 and CD80) concomitantly with an increase in M2-associated ones (CD163 and CD206) (84). Along 
these lines, RvD2 prevents cigarette smoke extract (CSE)induced M1 polarization (CD80) and enhance M2 polarization (CD206) in monocyte-derived macrophages (56). Further evidence is suggestive for the existence of distinct lipid mediator profiles among different macrophage subtypes; M2 macrophages (CD163 and CD206) have been found to generate higher levels of SPMs, whereas M1 macrophages (CD68 and CD86) have been shown to produce elevated amounts of prostanoids and leukotrienes, thereby promoting the amplification of inflammation (85). Moreover, the activating protein of ALOX5 enzyme was induced in M1, but not in M2 macrophages, while ALOX15 was upregulated in M2, but not in M1, macrophages (85). It was recently found that macrophages produce also a family of DHA-derived SPM molecules, named maresins (MaRs) with autocrine actions (79). In vitro treatment with the bioactive SPM, 13,14-epoxy-MaR resulted in higher levels of MaR1 in M2 macrophages (CD163 and CD206) compared to M1 macrophages (CD54 and CD80), suggesting a higher predisposition towards the proresolving activity of this macrophage subtype (84). Remarkably, in vivo administration of RvD2 favored a macrophage phenotype $\left(\right.$ Ly $\left.6 C^{\text {lo }}\right)$, with unique pro-resolving properties, stimulating inflammation resolution and tissue (muscle) regeneration (86).

A key phenotypic characteristic of mouse pro-inflammatory macrophages (M1) is the excessive production of nitric oxide (NO) $(10,14)$ and other reactive species, as part of their oxidative burst anti-microbial mechanisms. Maresin-like lipid mediator 14S,21R-dihydroxy-docosahexaenoic acid was shown to reduce the hyperglycemia-induced ROS production by macrophages (87) and modulate the ability of mesenchymal stem cells to induce ROS generation from macrophages under ischemia/reperfusion conditions (88). LXs and their aspirin-triggered epimers were shown to interfere with reactive species production by various cell types including monocytes and neutrophils $(89,90)$. Given the potential relationship of oxidative stress with the pathogenesis of COVID-19 (91), SPMs may have further beneficial effects in the current pandemic.

Thus, and in line with the previous functional effects mentioned above, a growing body of evidence points to the SPMs-induced phenotypic shift of macrophages towards an antiinflammatory and pro-resolving subtype.

\section{SPMS IN REGULATING THE VIRAL CELL- INFECTION AND TISSUE FIBROSIS}

Apart from their regulatory role in monocytes and macrophages, SPMs may also exhibit direct anti-viral effects, further enhancing their therapeutic potentials in complementing current anti-viral strategies. Stimulation of isolated macrophages from volunteers with CF with $\mathrm{S} 1$ protein from SARS-CoV2 triggered the biosynthesis of $\mathrm{RvD} 1$ pointing to the activation of proresolving signals in response to SARS-CoV2 (71). Activation of TLR7, an important pattern recognition receptor of viral RNA as noted above, is also known to stimulate SPMs production (92).
Of note, a direct mechanistic effect of SPMs was also shown; protectin 1 interacts with the RNA replication machinery of influenza virus and inhibits viral RNA nuclear export, thereby suppressing the pathogenicity of the virus (93). Although similar protective events have not yet addressed for SARS-CoV2, given the similarities in the replication of RNA viruses, one cannot exclude the high possibility of such speculation. Of note, 17HDHA has been shown to increase antibody production (IgM, IgG) by enhancing $\mathrm{B}$ cell differentiation towards an antibodysecreting B cell phenotype (CD80, CD86) in H1N1 influenza infection in mice (94) alluding to the regulatory effects of SPMs in the humoral immune response against viruses.

As noted above, advanced COVID-19 is also characterized by excessive fibrosis in lungs and other organs, most probably as a consequence of failures in the regulation of inflammation; unresolved inflammation has been closely related to tissue fibrosis and impaired organ function (95). Proinflammatory eicosanoids, such as leukotrienes, promote tissue fibrosis, whereas AT-LXs and synthetic benzo-LXA 4 analog have been shown to reduce bleomycin-induced pulmonary fibrosis and renal fibrosis respectively $(96,97)$. SPMs protective effect against fibrosis, is mediated at least in part by the reduction in collagen deposition (97). Thus, SPMs may also hold additional therapeutic modalities against severe COVID-19 manifestations, such as multiple organ fibrosis.

\section{CONCLUSIONS AND PERSPECTIVES}

Corona virus pandemic of 2019 has emerged as one of the biggest threats of our times, with outrageous health, social, economic and financial consequences globally. Since the identification of a cytokine storm in severe COVID-19 and the central role of pathogenic alveolar macrophage populations in the maintenance of hyperinflammation, characterized the severe disease, many current therapeutic research efforts have focused on antiinflammatory approaches. However, treating individual cytokines may not be sufficient. SPMs have the advantage of stimulating the physiological homeostatic processes towards the resolution of inflammation, acting directly on macrophages on various levels (Figure 1), without inducing systemic immunosuppression. Moreover, clinical experimentation has alluded to the safety of SPMs in humans. Exogenous administration of EPA and DHA (Lovaza) restored the endogenous SPMs levels in coronary artery patients, without any severe adverse effects being reported (80). Furthermore, SPMs may also hold beneficial effects against COVID-19, beyond regulating macrophages responses. Table 1 summarizes the immuno-regulatory functions of SPMs in relation to disease pathology, as discussed here. Interspecies differences in macrophage functions may well exist [discussed in (98)]; however many of the studies on SPMs addressed here, have used human primary macrophages. The importance of lipid mediators in COVID-19 progression is highlighted by studies showing a direct link between a lipidomic imbalance and disease severity. Even though SPMs are excessively 


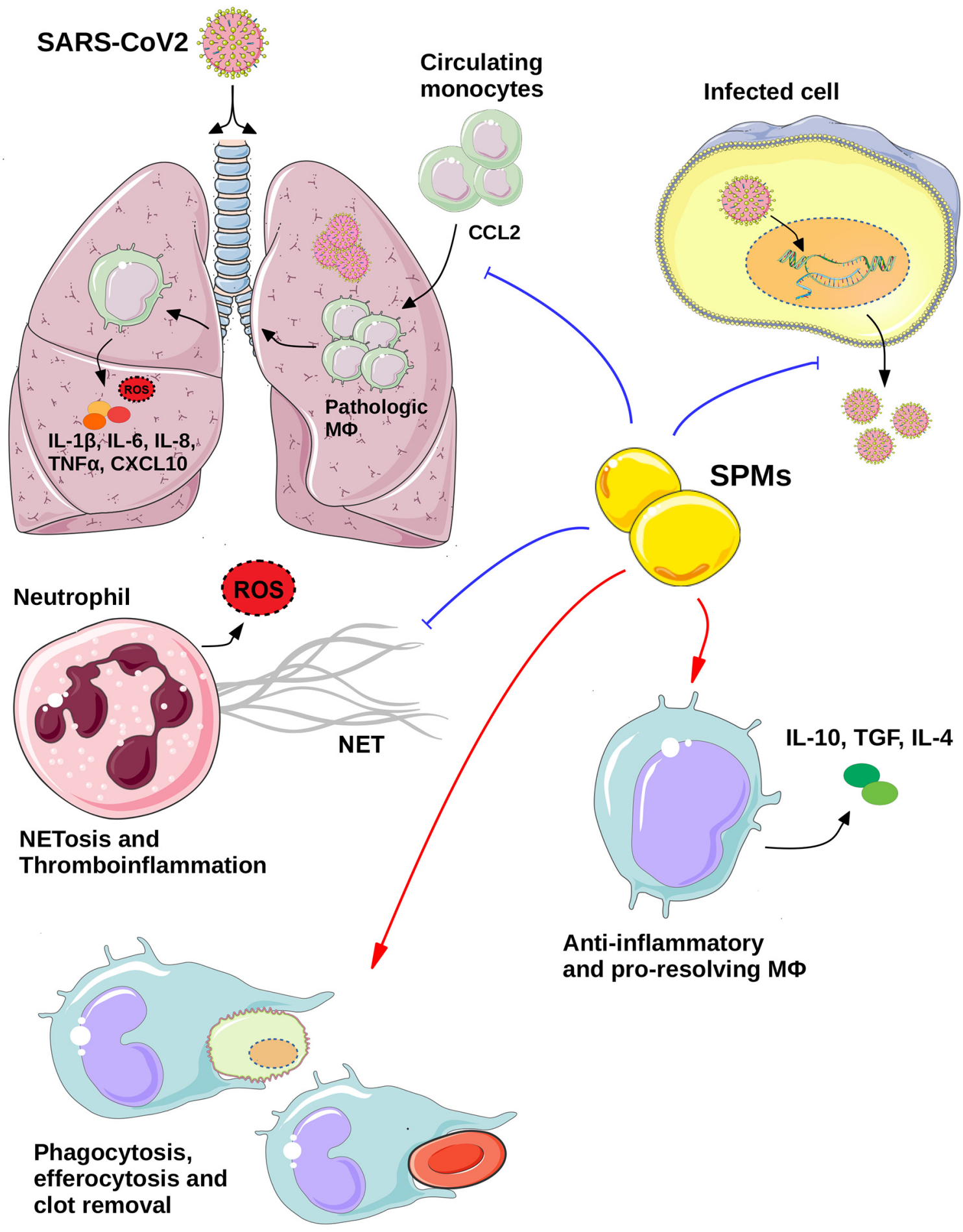

FIGURE 1 | The potential therapeutic role of SPMs in COVID-19. Specialized pro-resolving regulators (SPMs) mediate their anti-viral effects via directly inhibiting the viral replication and thus export of viral particles from infected cells and/or via regulating the functions of key immune cell types. SPMs act on neutrophils inhibiting NETosis and thrombroinflammation, while they regulate the macrophages responses on various levels including: inhibition of monocytes infiltration to the inflammed lungs, as well as, stimulation of homeostatic processes, via induction of efferocytosis, phagocytosis of debris and blood clots and anti-inflammatory and pro-resolving macrophages polarization. All these effects may eventually lead to the resolution of SARS-CoV2-induced inflammation and thus, potential management of COVID-19 pathologies. Blue lines indicate inhibition, whereas red arrows indicate induction. (Templates from Servier Medical ART, https://smart.servier.com/, were used to create this image) MФ, Macrophage; ROS, Reactive oxygen species. 
TABLE 1 | Immunoprotective actions of SPMs in relation to COVID-19 pathology.

\begin{tabular}{|c|c|c|c|}
\hline SPM & Cell-type/disease model & Mechanism of action & Reference \\
\hline \multicolumn{4}{|c|}{ Leukocytes adhesion, chemotaxis and migration } \\
\hline Resolvin E1 & $\begin{array}{l}\text { Human whole blood, intravital microscopy in } \\
\text { mice venules }\end{array}$ & $\begin{array}{l}\text { Downregulates leukocyte expression of adhesion molecules } \\
\text { Reduces leukocyte rolling ( } 40 \%) \text { on the endothelium }\end{array}$ & $\begin{array}{l}\text { Dona et al. } \\
(59)\end{array}$ \\
\hline AT- Resolvin D1 & $\begin{array}{l}\text { Bacterial and viral lung co-infection model in } \\
\text { mice }\end{array}$ & $\begin{array}{l}\text { Reduces infiltrating monocytes/macrophage numbers (CD11 } \mathrm{b}^{\mathrm{Hi}} \text {, } \\
\text { CD11 } \mathrm{c}^{\text {Low }} \text { ) }\end{array}$ & $\begin{array}{l}\text { Wang et al. } \\
(63)\end{array}$ \\
\hline Resolvin E1 & Human M1-like macrophages & $\begin{array}{l}\text { Reduces migration of M1-like macrophages (activated with LPS) } \\
\text { towards the chemotactic protein chemerin }\end{array}$ & $\begin{array}{l}\text { Herova et al } \\
(60)\end{array}$ \\
\hline $\begin{array}{l}\text { Resolvin D1 } \\
\text { Lipoxin } \mathrm{A}_{4}\end{array}$ & $\begin{array}{l}\text { In vitro microfluidic inflammation model based } \\
\text { on primary human neutrophil and monocytes }\end{array}$ & Suppress monocyte trafficking toward $\mathrm{LTB}_{4}$ & $\begin{array}{l}\text { Jones et al. } \\
(62)\end{array}$ \\
\hline \multicolumn{4}{|c|}{ Generation of cytokines and chemokines } \\
\hline Resolvin D1 & Human macrophages from cystic fibrosis & Reduce release of IL-8, MCP-1, MIP- $1 \alpha$ and CCL4 & Recchiuti \\
\hline Resolvin D2 & $\begin{array}{l}\text { individuals stimulated with S1 protein from } \\
\text { SARS-CoV2 }\end{array}$ & Upregulates expression of IL1RA & et al. (71) \\
\hline AT- Resolvin D3 & Human monocyte-derived macrophages & Decrease secretion of MIF, PAI-1 and CCL2 & Gilligan et al \\
\hline AT-Lipoxin $\mathrm{A}_{4}$ & & & $(66)$ \\
\hline Resolvin D1 & LPS-stimulated primary human monocytes & Inhibit secretion of TNF- $\alpha, I L-1 \beta, I L-8$ by activating the GSK3 $\beta$ & Gu et al. \\
\hline Resolvin D2 & & anti-inflammatory axis & $(65)$ \\
\hline \multicolumn{4}{|l|}{ Maresin 1} \\
\hline Resolvin D2 & $\begin{array}{l}\text { Alveolar macrophages from COPD and non- } \\
\text { COPD individuals }\end{array}$ & $\begin{array}{l}\text { Downregulate production of TNF- } \alpha \text { and IL-6, stimulate the } \\
\text { production of IL-10 and TGF- } \beta\end{array}$ & $\begin{array}{l}\text { Croasdell } \\
\text { et al. (56) }\end{array}$ \\
\hline $\begin{array}{l}\text { Resolvin peptide-containing } \\
\text { conjugates in tissue regeneration } \\
\text { (RCTRs) }\end{array}$ & Human monocyte-derived macrophages & $\begin{array}{l}\text { Downregulate CXCL9 and CCL7 Increase IL-10, IL-4, IL-11 and } \\
\text { TGF- } \alpha\end{array}$ & $\begin{array}{l}\text { De la Rosa } \\
\text { et al. (67) }\end{array}$ \\
\hline Resolvin D1 & Peritoneal macrophages in mice & Decreases TNF- $\alpha, \mathrm{IL}-6, \mathrm{CCL} 2$ and IL-1 $\beta$ & $\begin{array}{l}\text { Kain et al. } \\
(70)\end{array}$ \\
\hline \multicolumn{4}{|l|}{ Phagocytosis and efferocytosis } \\
\hline $\begin{array}{l}\text { Resolvin peptide-containing } \\
\text { conjugates in tissue regeneration } \\
\text { (RCTRs) }\end{array}$ & Human monocyte-derived macrophages & Stimulate phagocytosis and efferocytosis & $\begin{array}{l}\text { De la Rosa } \\
\text { et al. (67) }\end{array}$ \\
\hline Resolvin D2 & $\begin{array}{l}\text { Alveolar macrophages from COPD and non- } \\
\text { COPD individuals }\end{array}$ & Restore cigarette smoke-induced defects in phagocytosis & $\begin{array}{l}\text { Croasdell } \\
\text { et al. (56) }\end{array}$ \\
\hline $\begin{array}{l}\text { AT- Resolvin D3 } \\
\text { Resolvin D6 }\end{array}$ & Human monocyte-derived macrophages & $\begin{array}{l}\text { Upregulate macrophage phagocytosis of blood clots and promote } \\
\text { blood clot removal }\end{array}$ & $\begin{array}{l}\text { Elajami et al. } \\
\text { (80) }\end{array}$ \\
\hline
\end{tabular}

AT-Protectin D1

AT-Lipoxin $B_{4}$

\section{Macrophages polarization}

Maresin 1

Resolvin D1

Resolvin D2

13,14-epoxy-Maresin

Resolvin D2

\section{NETosis and thrombus resolution}

Resolvin D1

Resolvin D4

\section{Oxidative stress}

14S,21R-dihydroxy-

docosahexaenoic acid

14S,21R-dihydroxy-

docosahexaenoic acid

Lipoxin A4

AT 15-epi-LXA4

\section{Anti-viral effects}

Protectin 1

17- $\mathrm{HDHA}$
Abdominal aortic aneurysm model in mice

Deep vein thrombosis model in mice

Influenza in mice

Human monocyte-derived macrophages

Human monocyte-derived macrophages

Human monocyte-derived macrophages

Muscle-infiltrating macrophages from mice

Murine macrophages isolated by peritoneal lavage

Murine macrophage cell line (RAW264.7)

Human neutrophils and monocytes

H1N1 influenza infection in mice
Downregulate M1-associated markers (CD54 and CD80) and increase M2-associated markers (CD163 and CD206)

Prevents cigarette smoke extract (CSE)-induced M1 polarization (CD80) and enhance M2 polarization (CD206)

Increases the levels of SPMs in M2 macrophages (CD163 and CD206)

Increases Ly6C ${ }^{\text {lo }}$ macrophages and promotes regeneration

Suppresses NETosis markers,

IFN $\gamma$, IL-1 $\beta$, CXCL10 and MCP-1

Inhibits ionomycin-induced release of NETs and promotes

thrombus resolution

Improves diabetes-impaired pro-healing functions of macrophages Tian et al. by reducing hyperglycaemia-induced ROS production Modulate the ability of mesenchymal stem cells to induce ROS generation from macrophages under ischemia/reperfusion conditions

Reduce superoxide and peroxynitrite (ONOO-) formation Inhibit increase in intracellular diamino-fluorescein fluorescence (indicator of NO formation)

Interacts with the RNA replication machinery of influenza virus, inhibits viral RNA nuclear export and improves the survival Increase the number of antibody-secreting cells in vitro protecting against live $\mathrm{pH} 1 \mathrm{~N} 1$ influenza infection
Dalli et al.

(84)

Croasdell

et al. (56)

Dalli et al.

(84)

Giannakis et al. (86)

Spinosa

et al. (82)

Cherpokova

et al. (83)

(87)

Tian et al.

(88)

József et al.

(90)

Morita et al. (93)

Ramon et al. (94) 
TABLE 1 | Continued

\begin{tabular}{|c|c|c|c|}
\hline SPM & Cell-type/disease model & Mechanism of action & Reference \\
\hline AT- Resolvin D1 & $\begin{array}{l}\text { Co-infection with Streptococcus pneumoniae } \\
\text { and influenza A virus in mice }\end{array}$ & $\begin{array}{l}\text { Reduces severity of pneumonia by inhibiting excessive leukocyte } \\
\text { chemotaxis from the infected bronchioles to distal areas of the } \\
\text { lungs }\end{array}$ & $\begin{array}{l}\text { Wang et al. } \\
(63)\end{array}$ \\
\hline Resolvin E1 & HSV-1-induced stromal keratitis (SK) in mice & $\begin{array}{l}\text { Reduce the number of Th1,Th17 cells and neutrophils infiltrating } \\
\text { the cornea }\end{array}$ & $\begin{array}{l}\text { Rajasagi } \\
\text { et al. (61) }\end{array}$ \\
\hline \multicolumn{4}{|l|}{ Anti-fibrotic effects } \\
\hline AT-synthetic lipoxin analog & Pulmonary fibrosis in mice & $\begin{array}{l}\text { Reduces inflammation and matrix deposition } \\
\text { Inhibits bleomycin-induced pulmonary fibrosis and renal fibrosis }\end{array}$ & $\begin{array}{l}\text { Martins et al. } \\
(96)\end{array}$ \\
\hline $\begin{array}{l}\text { Lipoxin A4 } \\
\text { synthetic benzo- Lipoxin A4 }\end{array}$ & Early renal fibrosis in mice & $\begin{array}{l}\text { Decreases collagen deposition and inhibits progression of renal } \\
\text { fibrosis }\end{array}$ & $\begin{array}{l}\text { Börgeson } \\
\text { et al. (97) }\end{array}$ \\
\hline
\end{tabular}

attractive as potential therapeutic candidates in COVID-19 (99102), either exogenously administered or by inducing their endogenous production in patients, the relevant clinical evidence is still lacking, yet highly anticipated.

\section{AUTHOR CONTRIBUTIONS}

$\mathrm{MB}$ performed the literature research and wrote the manuscript. EP contributed to manuscript writing and formatting and provided critical scientific input. PC conceived the idea, designed the lay out, and wrote and reviewed the manuscript. All authors contributed to the article and approved the submitted version.

\section{FUNDING}

This research was supported by funds provided by the SouthEastern Norway Regional Health Authority, Project Number 2019059 to PC. Supported in part by USPHS grant K08DE027119 to EP from the National Institute of Dental and Craniofacial Research (NIDCR).

\section{REFERENCES}

1. Zhu N, Zhang D, Wang W, Li X, Yang B, Song J, et al. A Novel Coronavirus from Patients with Pneumonia in China, 2019. N Engl J Med (2020) 382 (8):727-33. doi: 10.1056/NEJMoa2001017

2. Hadjadj J, Yatim N, Barnabei L, Corneau A, Boussier J, Smith N, et al. Impaired type I interferon activity and inflammatory responses in severe COVID-19 patients. Science (2020) 369(6504):718-24. doi: 10.1126/ science.abc6027

3. Blanco-Melo D, Nilsson-Payant BE, Liu W-C, Uhl S, Hoagland D, Møller R, et al. Imbalanced Host Response to SARS-CoV-2 Drives Development of COVID-19. Cell (2020) 181(5):1036-45.e9. doi: 10.1016/j.cell.2020.04.026

4. Wu Z, McGoogan JM. Characteristics of and Important Lessons From the Coronavirus Disease 2019 (COVID-19) Outbreak in China: Summary of a Report of 72314 Cases From the Chinese Center for Disease Control and Prevention. JAMA (2020) 323(13):1239-42. doi: 10.1001/jama.2020.2648

5. Merad M, Martin JC. Pathological inflammation in patients with COVID19: a key role for monocytes and macrophages. Nat Rev Immunol (2020) 20 (6):355-62. doi: 10.1038/s41577-020-0331-4

6. Chawla A. Control of macrophage activation and function by PPARs. Circ Res (2010) 106(10):1559-69. doi: 10.1161/CIRCRESAHA.110.216523

7. Franken L, Schiwon M, Kurts C. Macrophages: sentinels and regulators of the immune system. Cell Microbiol (2016) 18(4):475-87. doi: 10.1111/ cmi. 12580

8. Netea MG, Joosten LA, Latz E, Mills KH, Natoli G, Stunnenberg HG, et al. Trained immunity: A program of innate immune memory in health and disease. Science (2016) 352(6284):aaf1098. doi: 10.1126/ science.aaf 1098

9. Mantovani A, Netea MG. Trained Innate Immunity, Epigenetics, and Covid19. N Engl J Med (2020) 383(11):1078-80. doi: 10.1056/NEJMcibr2011679

10. Mills CD, Kincaid K, Alt JM, Heilman MJ, Hill AM. M-1/M-2 macrophages and the Th1/Th2 paradigm. J Immunol (2000) 164(12):6166-73. doi: 10.4049/jimmunol.164.12.6166

11. Mantovani A, Sozzani S, Locati M, Allavena P, Sica A. Macrophage polarization: tumor-associated macrophages as a paradigm for polarized M2 mononuclear phagocytes. Trends Immunol (2002) 23(11):549-55. doi: 10.1016/S1471-4906(02)02302-5

12. Biswas SK, Mantovani A. Macrophage plasticity and interaction with lymphocyte subsets: cancer as a paradigm. Nat Immunol (2010) 11 (10):889-96. doi: 10.1038/ni.1937

13. Martinez FO, Gordon S. The M1 and M2 paradigm of macrophage activation: time for reassessment. F1000Prime Rep (2014) 6:13. doi: 10.12703/P6-13

14. Murray PJ, Allen JE, Biswas SK, Fisher EA, Gilroy DW, Goerdt S, et al. Macrophage activation and polarization: nomenclature and experimental guidelines. Immunity (2014) 41(1):14-20. doi: 10.1016/j.immuni.2014.06.008

15. Jensen S, Thomsen AR. Sensing of RNA viruses: a review of innate immune receptors involved in recognizing RNA virus invasion. J Virol (2012) 86 (6):2900-10. doi: 10.1128/JVI.05738-11

16. Moreno-Eutimio MA, López-Macías C, Pastelin-Palacios R. Bioinformatic analysis and identification of single-stranded RNA sequences recognized by TLR7/8 in the SARS-CoV-2, SARS-CoV, and MERS-CoV genomes. Microbes Infect (2020) 22(4-5):226-9. doi: 10.1016/j.micinf.2020.04.009

17. van der Made CI, Simons A, Schuurs-Hoeijmakers J, van den Heuvel G, Mantere T, Kersten S, et al. Presence of Genetic Variants Among Young Men With Severe COVID-19. JAMA (2020) 324(7):1-11. doi: 10.1001/ jama.2020.13719

18. Jamilloux Y, Henry T, Belot A, Viel S, Fauter M, El Jammal T, et al. Should we stimulate or suppress immune responses in COVID-19? Cytokine and anti-cytokine interventions. Autoimmun Rev (2020) 19(7):102567. doi: 10.1016/j.autrev.2020.102567

19. Zhou F, Yu T, Du R, Fan G, Liu Y, Liu Z, et al. Clinical course and risk factors for mortality of adult inpatients with COVID-19 in Wuhan, China: a retrospective cohort study. Lancet (2020) 395(10229):1054-62. doi: 10.1016/S0140-6736(20)30566-3

20. Fu L, Wang B, Yuan T, Chen X, Ao Y, Fitzpatrick T, et al. Clinical characteristics of coronavirus disease 2019 (COVID-19) in China: A 
systematic review and meta-analysis. J Infect (2020) 80(6):656-65. doi: 10.1016/j.jinf.2020.03.041

21. Huang C, Wang Y, Li X, Ren L, Zhao J, Hu Y, et al. Clinical features of patients infected with 2019 novel coronavirus in Wuhan, China. Lancet (2020) 395(10223):497-506. doi: 10.1016/S0140-6736(20)30183-5

22. Perlman S, Netland J. Coronaviruses post-SARS: update on replication and pathogenesis. Nat Rev Microbiol (2009) 7(6):439-50. doi: 10.1038/ nrmicro2147

23. Channappanavar R, Fehr AR, Vijay R, Mack M, Zhao J, Meyerholz DK, et al. Dysregulated Type I Interferon and Inflammatory Monocyte-Macrophage Responses Cause Lethal Pneumonia in SARS-CoV-Infected Mice. Cell Host Microbe (2016) 19(2):181-93. doi: 10.1016/j.chom.2016.01.007

24. Brunetta E, Folci M, Bottazzi B, De Santis M, Gritti G, Protti A, et al. Macrophage expression and prognostic significance of the long pentraxin PTX3 in COVID-19. Nat Immunol (2020) 22(1):19-24. doi: 10.1101/ 2020.06.26.20139923

25. Vega MA, Simón-Fuentes M, González de la Aleja A, Nieto C, Colmenares M, Herrero C, et al. MAFB and MAF Transcription Factors as Macrophage Checkpoints for COVID-19 Severity. Front Immunol (2020) 11(2958) 603507. doi: 10.3389/fimmu.2020.603507

26. Pagliaro P. Is macrophages heterogeneity important in determining COVID-19 lethality? Med Hypotheses (2020) 143:110073. doi: 10.1016/ j.mehy.2020.110073

27. Castagnoli R, Votto M, Licari A, Brambilla I, Bruno R, Perlini S, et al. Severe Acute Respiratory Syndrome Coronavirus 2 (SARS-CoV-2) Infection in Children and Adolescents: A Systematic Review. JAMA Pediatr (2020) 174 (9):882-9. doi: 10.1001/jamapediatrics.2020.1467

28. Chua RL, Lukassen S, Trump S, Hennig BP, Wendisch D, Pott F, et al. COVID-19 severity correlates with airway epithelium-immune cell interactions identified by single-cell analysis. Nat Biotechnol (2020) 38 (8):970-9. doi: 10.1038/s41587-020-0602-4

29. Liao M, Liu Y, Yuan J, Wen Y, Xu G, Zhao J, et al. Single-cell landscape of bronchoalveolar immune cells in patients with COVID-19. Nat Med (2020) 26(6):842-4. doi: 10.1038/s41591-020-0901-9

30. Yang Y, Shen C, Li J, Yuan J, Wei J, Huang F, et al. Plasma IP-10 and MCP-3 levels are highly associated with disease severity and predict the progression of COVID-19. J Allergy Clin Immunol (2020) 146(1):119-27.e4. doi: 10.1016/j.jaci.2020.04.027

31. Roumier M, Paule R, Groh M, Vallee A, Ackermann F. Interleukin-6 blockade for severe COVID-19. medRxiv (2020). 2020.04.20.20061861. doi: 10.1101/2020.04.20.20061861

32. McGonagle D, Sharif K, O'Regan A, Bridgewood C. The Role of Cytokines including Interleukin-6 in COVID-19 induced Pneumonia and Macrophage Activation Syndrome-Like Disease. Autoimmun Rev (2020) 19(6):102537-. doi: 10.1016/j.autrev.2020.102537

33. Tang Y, Liu J, Zhang D, Xu Z, Ji J, Wen C. Cytokine Storm in COVID-19: The Current Evidence and Treatment Strategies. Front Immunol (2020) 11:1708-. doi: 10.3389/fimmu.2020.01708

34. Serhan CN, Levy BD. Resolvins in inflammation: emergence of the proresolving superfamily of mediators. J Clin Invest (2018) 128(7):2657-69. doi: 10.1172/JCI97943

35. Chiang N, Serhan CN. Cell-cell interaction in the transcellular biosynthesis of novel omega-3-derived lipid mediators. Methods Mol Biol (2006) 341:227-50. doi: 10.1385/1-59745-113-4:227

36. Levy BD, Clish CB, Schmidt B, Gronert K, Serhan CN. Lipid mediator class switching during acute inflammation: signals in resolution. Nat Immunol (2001) 2(7):612-9. doi: 10.1038/89759

37. Kantarci A, Van Dyke TE. Lipoxins in chronic inflammation. Crit Rev Oral Biol Med (2003) 14(1):4-12. doi: 10.1177/154411130301400102

38. Chiang N, Bermudez EA, Ridker PM, Hurwitz S, Serhan CN. Aspirin triggers antiinflammatory 15-epi-lipoxin A4 and inhibits thromboxane in a randomized human trial. Proc Natl Acad Sci U S A (2004) 101(42):1517883. doi: $10.1073 /$ pnas. 0405445101

39. Arita $\mathrm{M}$, Clish $\mathrm{CB}$, Serhan $\mathrm{CN}$. The contributions of aspirin and microbial oxygenase to the biosynthesis of anti-inflammatory resolvins: novel oxygenase products from omega-3 polyunsaturated fatty acids. Biochem Biophys Res Commun (2005) 338(1):149-57. doi: 10.1016/j.bbrc.2005. 07.181
40. Serhan CN, Clish CB, Brannon J, Colgan SP, Gronert K, Chiang N. Antimicroinflammatory lipid signals generated from dietary N-3 fatty acids via cyclooxygenase- 2 and transcellular processing: a novel mechanism for NSAID and N-3 PUFA therapeutic actions. J Physiol Pharmacol (2000) 51 (4 Pt 1):643-54.

41. Chiang N, Serhan Charles N. Specialized pro-resolving mediator network: an update on production and actions. Essays Biochem (2020) 64(3):443-62. doi: 10.1042/EBC20200018

42. Hafner AK, Kahnt AS, Steinhilber D. Beyond leukotriene formation-The noncanonical functions of 5-lipoxygenase. Prostaglandins Other Lipid Mediat (2019) 142:24-32. doi: 10.1016/j.prostaglandins.2019.03.003

43. Fredman G, Hellmann J, Proto JD, Kuriakose G, Colas RA, Dorweiler B, et al. An imbalance between specialized pro-resolving lipid mediators and pro-inflammatory leukotrienes promotes instability of atherosclerotic plaques. Nat Commun (2016) 7:12859. doi: 10.1038/ncomms12859

44. Fredman G, Ozcan L, Spolitu S, Hellmann J, Spite M, Backs J, et al. Resolvin D1 limits 5-lipoxygenase nuclear localization and leukotriene B4 synthesis by inhibiting a calcium-activated kinase pathway. Proc Natl Acad Sci U S A (2014) 111(40):14530-5. doi: 10.1073/pnas.1410851111

45. Li Y, Dalli J, Chiang N, Baron RM, Quintana C, Serhan CN. Plasticity of leukocytic exudates in resolving acute inflammation is regulated by MicroRNA and proresolving mediators. Immunity (2013) 39(5):885-98. doi: 10.1016/j.immuni.2013.10.011

46. Serhan CN, Chiang N, Van Dyke TE. Resolving inflammation: dual antiinflammatory and pro-resolution lipid mediators. Nat Rev Immunol (2008) 8 (5):349-61. doi: 10.1038/nri2294

47. Filep JG. Biasing the lipoxin A4/formyl peptide receptor 2 pushes inflammatory resolution. Proc Natl Acad Sci U S A (2013) 110(45):180334. doi: 10.1073/pnas.1317798110

48. Li H, Xiang X, Ren H, Xu L, Zhao L, Chen X, et al. Serum Amyloid A is a biomarker of severe Coronavirus Disease and poor prognosis. J Infect (2020) 80(6):646-55. doi: 10.1016/j.jinf.2020.03.035

49. Bozinovski S, Uddin M, Vlahos R, Thompson M, McQualter JL, Merritt AS, et al. Serum amyloid A opposes lipoxin $\mathrm{A}_{4}$ to mediate glucocorticoid refractory lung inflammation in chronic obstructive pulmonary disease. Proc Natl Acad Sci U S A (2012) 109(3):935-40. doi: 10.1073/ pnas. 1109382109

50. Sorokin AV, Karathanasis SK, Yang ZH, Freeman L, Kotani K, Remaley AT. COVID-19-Associated dyslipidemia: Implications for mechanism of impaired resolution and novel therapeutic approaches. FASEB J (2020) 34 (8):9843-53. doi: 10.1096/fj.202001451

51. Schwarz B, Sharma L, Roberts L, Peng X, Bermejo S, Leighton I, et al. Cutting Edge: Severe SARS-CoV-2 Infection in Humans Is Defined by a Shift in the Serum Lipidome, Resulting in Dysregulation of Eicosanoid Immune Mediators. J Immunol (2020) 206(2):329-34. doi: 10.21203/ rs.3.rs-42999/v1

52. Tam VC. Lipidomic profiling of bioactive lipids by mass spectrometry during microbial infections. Semin Immunol (2013) 25(3):240-8. doi: 10.1016/j.smim.2013.08.006

53. Martínez-Martínez AB, Torres-Perez E, Devanney N, Del Moral R, Johnson LA, Arbones-Mainar JM. Beyond the CNS: The many peripheral roles of APOE. Neurobiol Dis (2020) 138:104809. doi: 10.1016/j.nbd.2020.104809

54. Sorokin AV, Yang ZH, Vaisman BL, Thacker S, Yu ZX, Sampson M, et al. Addition of aspirin to a fish oil-rich diet decreases inflammation and atherosclerosis in ApoE-null mice. J Nutr Biochem (2016) 35:58-65. doi: 10.1016/j.jnutbio.2016.05.012

55. Neuhofer A, Zeyda M, Mascher D, Itariu BK, Murano I, Leitner L, et al. Impaired local production of proresolving lipid mediators in obesity and 17HDHA as a potential treatment for obesity-associated inflammation. Diabetes (2013) 62(6):1945-56. doi: 10.2337/db12-0828

56. Croasdell A, Thatcher TH, Kottmann RM, Colas RA, Dalli J, Serhan CN, et al. Resolvins attenuate inflammation and promote resolution in cigarette smoke-exposed human macrophages. Am J Physiol Lung Cell Mol Physiol (2015) 309(8):L888-901. doi: 10.1152/ajplung.00125.2015

57. Misharin AV, Morales-Nebreda L, Reyfman PA, Cuda CM, Walter JM, McQuattie-Pimentel AC, et al. Monocyte-derived alveolar macrophages drive lung fibrosis and persist in the lung over the life span. J Exp Med (2017) 214(8):2387-404. doi: 10.1084/jem.20162152 
58. Aran D, Looney AP, Liu L, Wu E, Fong V, Hsu A, et al. Reference-based analysis of lung single-cell sequencing reveals a transitional profibrotic macrophage. Nat Immunol (2019) 20(2):163-72. doi: 10.1038/s41590-018-0276-y

59. Dona M, Fredman G, Schwab JM, Chiang N, Arita M, Goodarzi A, et al. Resolvin E1, an EPA-derived mediator in whole blood, selectively counterregulates leukocytes and platelets. Blood (2008) 112(3):848-55. doi: 10.1182/blood-2007-11-122598

60. Herová M, Schmid M, Gemperle C, Hersberger M. ChemR23, the receptor for chemerin and resolvin E1, is expressed and functional on M1 but not on M2 macrophages. J Immunol (2015) 194(5):2330-7. doi: 10.4049/ jimmunol.1402166

61. Rajasagi NK, Reddy PBJ, Suryawanshi A, Mulik S, Gjorstrup P, Rouse BT. Controlling Herpes Simplex Virus-Induced Ocular Inflammatory Lesions with the Lipid-Derived Mediator Resolvin E1. J Immunol (2011) 186 (3):1735-46. doi: 10.4049/jimmunol.1003456

62. Jones CN, Dalli J, Dimisko L, Wong E, Serhan CN, Irimia D. Microfluidic chambers for monitoring leukocyte trafficking and humanized nanoproresolving medicines interactions. Proc Natl Acad Sci U S A (2012) 109 (50):20560-5. doi: 10.1073/pnas.1210269109

63. Wang H, Anthony D, Yatmaz S, Wijburg O, Satzke C, Levy B, et al. Aspirintriggered resolvin D1 reduces pneumococcal lung infection and inflammation in a viral and bacterial coinfection pneumonia model. Clin Sci (Lond) (2017) 131(18):2347-62. doi: 10.1042/CS20171006

64. Dalli J, Serhan CN. Pro-Resolving Mediators in Regulating and Conferring Macrophage Function. Front Immunol (2017) 8:1400. doi: 10.3389/ fimmu.2017.01400

65. Gu Z, Lamont GJ, Lamont RJ, Uriarte SM, Wang H, Scott DA. Resolvin D1, resolvin D2 and maresin 1 activate the GSK3 $\beta$ anti-inflammatory axis in TLR4-engaged human monocytes. Innate Immun (2016) 22(3):186-95. doi: $10.1177 / 1753425916628618$

66. Gilligan MM, Gartung A, Sulciner ML, Norris PC, Sukhatme VP, Bielenberg $\mathrm{DR}$, et al. Aspirin-triggered proresolving mediators stimulate resolution in cancer. Proc Natl Acad Sci U S A (2019) 116(13):6292-7. doi: 10.1073/ pnas. 1804000116

67. de la Rosa X, Norris PC, Chiang N, Rodriguez AR, Spur BW, Serhan CN. Identification and Complete Stereochemical Assignments of the New Resolvin Conjugates in Tissue Regeneration in Human Tissues that Stimulate Proresolving Phagocyte Functions and Tissue Regeneration. Am J Pathol (2018) 188(4):950-66. doi: 10.1016/j.ajpath.2018.01.004

68. Werz O, Gerstmeier J, Libreros S, De la Rosa X, Werner M, Norris PC, et al. Human macrophages differentially produce specific resolvin or leukotriene signals that depend on bacterial pathogenicity. Nat Commun (2018) 9(1):59. doi: 10.1038/s41467-017-02538-5

69. Wang JC, Strichartz GR. Prevention of Chronic Post-Thoracotomy Pain in Rats By Intrathecal Resolvin D1 and D2: Effectiveness of Perioperative and Delayed Drug Delivery. J Pain (2017) 18(5):535-45. doi: 10.1016/ j.jpain.2016.12.012

70. Kain V, Halade GV. Immune responsive resolvin D1 programs peritoneal macrophages and cardiac fibroblast phenotypes in diversified metabolic microenvironment. J Cell Physiol (2019) 234(4):3910-20. doi: 10.1002/ jcp. 27165

71. Recchiuti A, Patruno S, Mattoscio D, Isopi E, Pomilio A, Lamolinara A, et al. Resolvin D1 and D2 reduce SARS-Cov-2-induced inflammation in cystic fibrosis macrophages. bioRxiv (2020). 2020.08.28.255463. doi: 10.1101/ 2020.08.28.255463

72. Kourtzelis I, Hajishengallis G, Chavakis T. Phagocytosis of Apoptotic Cells in Resolution of Inflammation. Front Immunol (2020) 11:553. doi: 10.3389/ fimmu.2020.00553

73. Doran AC, Yurdagul A Jr, Tabas I. Efferocytosis in health and disease. Nat Rev Immunol (2020) 20(4):254-67. doi: 10.1038/s41577-019-0240-6

74. Elliott MR, Koster KM, Murphy PS. Efferocytosis Signaling in the Regulation of Macrophage Inflammatory Responses. J Immunol (2017) 198(4):1387-94. doi: 10.4049/jimmunol.1601520

75. Serhan CN, Dalli J, Karamnov S, Choi A, Park CK, Xu ZZ, et al. Macrophage proresolving mediator maresin 1 stimulates tissue regeneration and controls pain. FASEB J (2012) 26(4):1755-65. doi: 10.1096/fj.11-201442

76. Godson C, Mitchell S, Harvey K, Petasis NA, Hogg N, Brady HR. Cutting Edge: Lipoxins Rapidly Stimulate Nonphlogistic Phagocytosis of Apoptotic
Neutrophils by Monocyte-Derived Macrophages. J Immunol (2000) 164 (4):1663-7. doi: 10.4049/jimmunol.164.4.1663

77. Norris PC, Libreros S, Serhan CN. Resolution metabolomes activated by hypoxic environment. Sci $A d v$ (2019) 5(10):eaax4895. doi: 10.1126/ sciadv.aax 4895

78. Dalli J, Serhan CN. Specific lipid mediator signatures of human phagocytes: microparticles stimulate macrophage efferocytosis and pro-resolving mediators. Blood (2012) 120(15):e60-72. doi: 10.1182/blood-2012-04423525

79. Serhan CN, Yang R, Martinod K, Kasuga K, Pillai PS, Porter TF, et al. Maresins: novel macrophage mediators with potent antiinflammatory and proresolving actions. J Exp Med (2009) 206(1):15-23. doi: 10.1084/ jem. 20081880

80. Elajami TK, Colas RA, Dalli J, Chiang N, Serhan CN, Welty FK. Specialized proresolving lipid mediators in patients with coronary artery disease and their potential for clot remodeling. FASEB J (2016) 30(8):2792-801. doi: 10.1096/fj.201500155R

81. Arcanjo A, Logullo J, Menezes CCB, de Souza Carvalho Giangiarulo TC, Dos Reis MC, de Castro GMM, et al. The emerging role of neutrophil extracellular traps in severe acute respiratory syndrome coronavirus 2 (COVID-19). Sci Rep (2020) 10(1):19630. doi: 10.21203/rs.3.rs-40461/v1

82. Spinosa M, Su G, Salmon MD, Lu G, Cullen JM, Fashandi AZ, et al. Resolvin D1 decreases abdominal aortic aneurysm formation by inhibiting NETosis in a mouse model. J Vasc Surg (2018) 68(6s):93s-103s. doi: 10.1016/ j.jvs.2018.05.253

83. Cherpokova D, Jouvene CC, Libreros S, DeRoo EP, Chu L, de la Rosa X, et al. Resolvin D4 attenuates the severity of pathological thrombosis in mice. Blood (2019) 134(17):1458-68. doi: 10.1182/blood.2018886317

84. Dalli J, Zhu M, Vlasenko NA, Deng B, Haeggström JZ, Petasis NA, et al. The novel 13S,14S-epoxy-maresin is converted by human macrophages to maresin 1 (MaR1), inhibits leukotriene A4 hydrolase (LTA4H), and shifts macrophage phenotype. FASEB J (2013) 27(7):2573-83. doi: 10.1096/fj.13227728

85. Lim CS, Porter DW, Orandle MS, Green BJ, Barnes MA, Croston TL, et al. Resolution of Pulmonary Inflammation Induced by Carbon Nanotubes and Fullerenes in Mice: Role of Macrophage Polarization. Front Immunol (2020) 11:1186-. doi: 10.3389/fimmu.2020.01186

86. Giannakis N, Sansbury BE, Patsalos A, Hays TT, Riley CO, Han X, et al. Dynamic changes to lipid mediators support transitions among macrophage subtypes during muscle regeneration. Nat Immunol (2019) 20(5):626-36. doi: 10.1038/s41590-019-0356-7

87. Tian H, Lu Y, Shah SP, Hong S. Autacoid 14S,21R-DihydroxyDocosahexaenoic Acid Counteracts Diabetic Impairment of Macrophage Prohealing Functions. Am J Pathol (2011) 179(4):1780-91. doi: 10.1016/ j.ajpath.2011.06.026

88. Tian H, Lu Y, Shah SP, Wang Q, Hong S. 14S,21R-dihydroxydocosahexaenoic acid treatment enhances mesenchymal stem cell amelioration of renal ischemia/reperfusion injury. Stem Cells Dev (2012) 21(7):1187-99. doi: 10.1089/scd.2011.0220

89. Leuti A, Maccarrone M, Chiurchiù V. Proresolving Lipid Mediators: Endogenous Modulators of Oxidative Stress. Oxid Med Cell Longev (2019) 2019:8107265. doi: 10.1155/2019/8107265

90. József L, Zouki C, Petasis NA, Serhan CN, Filep JG. Lipoxin A4 and aspirin-triggered 15-epi-lipoxin A4 inhibit peroxynitrite formation, NFkappa B and AP-1 activation, and IL-8 gene expression in human leukocytes. Proc Natl Acad Sci U S A (2002) 99(20):13266-71. doi: 10.1073/pnas.202296999

91. Cecchini R, Cecchini AL. SARS-CoV-2 infection pathogenesis is related to oxidative stress as a response to aggression. Med Hypotheses (2020) 143:110102. doi: 10.1016/j.mehy.2020.110102

92. Koltsida O, Karamnov S, Pyrillou K, Vickery T, Chairakaki AD, Tamvakopoulos C, et al. Toll-like receptor 7 stimulates production of specialized pro-resolving lipid mediators and promotes resolution of airway inflammation. EMBO Mol Med (2013) 5(5):762-75. doi: 10.1002/emmm.201201891

93. Morita M, Kuba K, Ichikawa A, Nakayama M, Katahira J, Iwamoto R, et al. The lipid mediator protectin D1 inhibits influenza virus replication and improves severe influenza. Cell (2013) 153(1):112-25. doi: 10.1016/j.cell.2013. 02.027 
94. Ramon S, Baker SF, Sahler JM, Kim N, Feldsott EA, Serhan CN, et al. The specialized proresolving mediator 17-HDHA enhances the antibody-mediated immune response against influenza virus: a new class of adjuvant? J Immunol (Baltimore Md 1950) (2014) 193(12):6031-40. doi: 10.4049/jimmunol.1302795

95. Serhan CN, Chiang N, Dalli J. The resolution code of acute inflammation: Novel pro-resolving lipid mediators in resolution. Semin Immunol (2015) 27 (3):200-15. doi: 10.1016/j.smim.2015.03.004

96. Martins V, Valença SS, Farias-Filho FA, Molinaro R, Simões RL, Ferreira TP, et al. ATLa, an aspirin-triggered lipoxin A4 synthetic analog, prevents the inflammatory and fibrotic effects of bleomycin-induced pulmonary fibrosis. J Immunol (2009) 182(9):5374-81. doi: 10.4049/jimmunol.0802259

97. Börgeson E, Docherty NG, Murphy M, Rodgers K, Ryan A, O’Sullivan TP, et al. Lipoxin A4 and benzo-lipoxin A4 attenuate experimental renal fibrosis. FASEB J (2011) 25(9):2967-79. doi: 10.1096/fj.11-185017

98. Saas P, Chague C, Maraux M, Cherrier T. Toward the Characterization of Human Pro-Resolving Macrophages? Front Immunol (2020) 11(3005) 593300. doi: 10.3389/fimmu.2020.593300

99. Panigrahy D, Gilligan MM, Huang S, Gartung A, Cortés-Puch I, Sime PJ, et al. Inflammation resolution: a dual-pronged approach to averting cytokine storms in COVID-19? Cancer Metastasis Rev (2020) 39(2):337-40. doi: 10.1007/s10555-020-09889-4
100. Andreakos E, Papadaki M, Serhan CN. Dexamethasone, pro-resolving lipid mediators and resolution of inflammation in COVID-19. Allergy (2020). doi: 10.1111/all.14595

101. Regidor PA, Santos FG, Rizo JM, Egea FM. Pro resolving inflammatory effects of the lipid mediators of omega 3 fatty acids and its implication in SARS COVID-19. Med Hypotheses (2020) 145:110340. doi: 10.1016/ j.mehy.2020.110340

102. Regidor PA. Covid-19 management with inflammation resolving mediators? Perspectives and potential. Med Hypotheses (2020) 142:109813. doi: 10.1016/ j.mehy.2020.109813

Conflict of Interest: The authors declare that the research was conducted in the absence of any commercial or financial relationships that could be construed as a potential conflict of interest.

Copyright (C) 2021 Balta, Papathanasiou and Christopoulos. This is an open-access article distributed under the terms of the Creative Commons Attribution License (CC BY). The use, distribution or reproduction in other forums is permitted, provided the original author(s) and the copyright owner(s) are credited and that the original publication in this journal is cited, in accordance with accepted academic practice. No use, distribution or reproduction is permitted which does not comply with these terms. 